\title{
Diffuse galactic sub-MeV $\gamma$-ray excess from high-energy electrons
}

\author{
R. Schröder ${ }^{1}$, R. Schlickeiser ${ }^{1}$, and A. W. Strong ${ }^{2}$ \\ 11 Institut für Theoretische Physik, Lehrstuhl IV: Weltraum- und Astrophysik, Ruhr-Universität Bochum, 44780 Bochum, Germany \\ e-mail: rsch@tp4.rub.de \\ 2 Max-Planck-Institut für Extraterrestrische Physik, Postfach 1312, 85741 Garching, Germany
}

Received 22 July 2005 / Accepted 11 September 2005

\section{ABSTRACT}

Recent INTEGRAL observations have confirmed the existence of diffuse sub-MeV radiation from the inner Galaxy. We propose a new explanation for the origin of this diffuse component in terms of electrostatic bremsstrahlung radiation from high-energy electrons. This electrostatic bremsstrahlung interpretation requires a very flat power law energy spectrum of high-energy electrons in the inner Galaxy which is reasonable if many point sources with significant spectral dispersion contribute to the injection of high-energy electrons in the Galaxy. The electrostatic bremsstrahlung interpretation avoids the enormous source power problem that previous explanations by radiation from low-energy electrons have.

Key words. cosmic ray electrons - diffuse galactic gamma rays - radiation processes

\section{Introduction}

The OSSE (Kinzer et al. 1999) and COMPTEL (Strong et al. 1994) instruments on board of the Compton Gamma Ray Observatory established that the diffuse galactic gamma ray continuum emission extends down to photon energies below $100 \mathrm{keV}$. The truly diffuse origin of this radiation has been proven by correlated SIGMA measurements to estimate the galactic point source contribution and the analysis of the GINGA meassurements of the galactic ridge emission at much lower energies (Yamasaki et al. 1997). Recent INTEGRAL observations (Strong et al. 2003; Lebrun et al. 2004; Strong et al. 2004,2005 ) indicated that besides the positronium continuum, dominating the emission between 200 and $400 \mathrm{keV}$, a second diffuse galactic continuum emission component is present with a flat power law photon number spectral index of -1.7 from $20 \mathrm{keV}$ up to $1 \mathrm{MeV}$.

In the past this sub-MeV gamma-ray emission has been interpreted as nonthermal electron bremsstrahlung radiation from low-energy electrons (Skibo \& Ramaty 1993; Skibo et al. 1996; Schlickeiser 1997; Dogiel et al. 2002) in the interstellar medium. As the main energy loss processes of such low-energy electrons are Coulomb and ionization interactions, the electrons nonthermal bremsstrahlung efficiency is small, so that this interpretation requires large amounts of low-energy interstellar electrons. Attributing these to cosmic ray electron sources, Skibo \& Ramaty (1993) and Skibo et al. (1996) estimate that integrated over the whole Galaxy a source power of $\sim(1-10) \times 10^{42} \mathrm{erg} \mathrm{s}^{-1}$ in these low-energy $(<10 \mathrm{MeV})$ electrons is required to maintain these electrons against the severe
Coulomb and ionization losses. This power exceeds the power supplied to the nuclear cosmic ray component by at least two orders an order of magnitude, and represents a serious problem for the general problem of the origin of cosmic rays. It is the purpose of this work to provide an alternative interpretation of the Compton observatory and INTEGRAL sub-MeV measurements. We show that the sub-MeV diffuse gamma-ray emission can be explained by the electrostatic bremsstrahlung radiation from high-energy electrons which is inverse Compton scattering of electrostatic plasma waves into transverse electromagnetic photons. This electrostatic bremsstrahlung interpretation avoids the enormous source power problem of the radiating electrons, and relies on the existence of interstellar electrostatic plasma waves generated by the interaction of supernova outflows with the ambient interstellar medium.

\section{Synchrotron and electrostatic bremsstrahlung from high-energy cosmic-ray electrons}

\subsection{High energy cosmic ray electrons}

According to current understanding high-energy $\left(\gamma_{\mathrm{e}}>10^{4}\right)$ galactic cosmic ray electrons (negatrons and positrons) consist of two distinct populations: primary and secondary electrons. Primary cosmic ray electrons are accelerated in the same sources as high-energy cosmic ray hadrons whereas secondary electrons result from inelastic hadron-hadron collisions of cosmic ray hadrons in the interstellar medium. The equilibrium power law energy spectrum of secondary electrons then is very steep $\left(\propto \gamma_{\mathrm{e}}^{-3.7}\right)$ because the secondaries production 
spectrum $\left(\propto \gamma_{\mathrm{e}}^{-2.7}\right)$ reflecting the cosmic ray hadron equilibrium spectrum is steepened by radiative synchrotron and inverse Compton losses. Because of this steep spectrum secondary electrons cannot produce the sub-MeV gamma-ray emission (Mörsberger \& Schlickeiser 1997).

The interstellar cosmic ray primary electron spectrum results from the competition between injection from many discrete sources as shell-type supernova remnants, diffusive propagation in the interstellar medium and radiative losses. A number of authors (Brecher \& Burbidge 1972; Pohl \& Esposito 1998; Strong et al. 2000; Büsching et al. 2001; Büsching et al. 2005) have demonstrated that in this case the energy spectra of primary electrons and hadrons vary significantly over the galaxy and may be much harder than locally observed. The reasons are twofold:

1) the superposition of many power laws with dispersion in individual spectral index values does not produce a simple power law (Brecher \& Burbidge 1972) at all energies. At high energies it flattens due to the dominance of those point sources with the flattest spectral index;

2) at high energies the radiative loss times of electrons are extremely short. As the bulk streaming speed of cosmic rays is limited to sub-Alfvenic values they penetrate not very far from their sources.

In the following we assume that the line-of-sight averaged cosmic-ray electron differential number density in the inner Galaxy is given by

$N(\gamma)=N_{0} \gamma^{-s} \exp \left(-\gamma / \gamma_{0}\right)$

where the spectral index $s$ can be as small as 1.5 which is compatible with the smallest observed synchrotron radiation spectral index of shell-type supernova remnants in the Galaxy (Clark \& Caswell 1976; Göbel et al. 1981).

\subsection{Electrostatic bremsstrahlung}

Electrostatic bremsstrahlung, i.e. the inverse Compton scattering of electrostatic plasma waves into transverse electromagnetic waves by relativistic electrons, is very similar to synchrotron radiation which is the inverse Compton scattering of the virtual photons of the magnetic field into tranverse electromagnetic waves by relativistic electrons in the Weizsäcker-Williams approach. Therefore its polarisation and emission properties are similar to that of synchrotron radiation (Chiuderi \& Veltri 1974; Windsor \& Kellogg 1974). Recently, one of us (Schlickeiser 2003) has proposed electrostatic bremsstrahlung as an alternative (to synchrotron radiation) nonthermal radiation process in jets of active galactic nuclei. Electrostatic bremsstrahlung becomes competitive to synchrotron radiation if the energy density in electrostatic waves $U_{\mathrm{L}}$ is of the same order as the magnetic field energy density $U_{B}$. Our interpretaion here indicates that this is indeed the case in the interstellar medium, as plenty of electrostatic plasma waves are generated by the interaction of the supernova remnant shock waves with the ambient interstellar medium. Modeling the interaction of the supernova shock waves with its ambient medium kinetically as a cloud of electron-proton plasma moving through the electron-proton interstellar medium (Pohl et al. 2002; Schlickeiser et al. 2002) indicates that the resulting energy density of electrostatic waves is

$U_{\mathrm{L}}=\frac{2}{3} n_{\mathrm{e}} m_{\mathrm{p}}\left\langle V_{\mathrm{s}}\right\rangle^{2} f$

where $n_{\mathrm{e}}$ denotes the interstellar electron density, $\left\langle V_{\mathrm{s}}\right\rangle$ the average supernova shock wave speed and $f$ the volume fraction of interstellar medium swept by supernova explosions. For the hot coronal phase $\left(f=0.8, n_{\mathrm{e}}=10^{-3} \mathrm{~cm}^{-3}\right)$ and $\left\langle V_{\mathrm{s}}\right\rangle=300 \mathrm{~km} \mathrm{~s}^{-1}$ Eq. (2) yields $U_{\mathrm{L}}=8.5 \times 10^{-13} \mathrm{erg} \mathrm{cm}^{-3}$, which is of the same order as the galactic magnetic field energy density $U_{B}=6.4 \times 10^{-13} \mathrm{erg} \mathrm{cm}^{-3}$ for a $4 \mu \mathrm{G}$ magnetic field strength.

\subsection{Intensities of sub-MeV radiation from electrostatic bremsstrahlung and synchrotron radiation}

In order to calculate the synchrotron and electrostatic bremsstrahlung radiation intensities we use the monochromatic approximations of the respective differential spectral powers (Schlickeiser 2003)

$$
\begin{aligned}
& p_{\text {synch }}(v, \gamma)=2 c \sigma_{\mathrm{T}} U_{B} \gamma^{2} \delta\left(v-v_{\mathrm{s}} \gamma^{2}\right), \\
& p_{\mathrm{eb}}(v, \gamma)=\frac{128}{27} c \sigma_{\mathrm{T}} U_{\mathrm{L}} \gamma^{2} \delta\left(v-v_{\mathrm{b}} \gamma^{2}\right),
\end{aligned}
$$

where $c$ denotes the speed of light, $\sigma_{\mathrm{T}}$ the Thomson cross section, $U_{B}=B^{2} / 8 \pi$ the magnetic field energy density and $U_{\mathrm{L}}$ the energy density in electrostatic plasma waves. $v_{\mathrm{s}}=$ $16.8(B / 4 \mu \mathrm{G}) \mathrm{Hz}$ and $\nu_{\mathrm{b}}=400\left(n_{\mathrm{e}} / 10^{-3} \mathrm{~cm}^{-3}\right)^{1 / 2} \mathrm{~Hz}$ are the characteristic synchrotron and electrostatic bremsstrahlung frequencies, respectively.

We then obtain for spontaneous synchrotron emission coefficient

$$
\begin{aligned}
j_{\text {synch }}(v) & =\frac{1}{4 \pi} \int_{1}^{\infty} \mathrm{d} \gamma p_{\text {synch }}(v, \gamma) N(\gamma) \\
& =\frac{c \sigma_{\mathrm{T}} U_{B} N_{0}}{4 \pi v_{\mathrm{s}}}\left(v / v_{\mathrm{s}}\right)^{(1-s) / 2} \exp \left(-\left(\frac{v}{v_{\mathrm{s}} \gamma_{0}^{2}}\right)^{1 / 2}\right)
\end{aligned}
$$

for frequencies $v>v_{\mathrm{s}}$. Likewise, the spontaneous electrostatic bremsstrahlung emission coefficient is

$$
\begin{aligned}
j_{\mathrm{eb}}(v) & =\frac{1}{4 \pi} \int_{1}^{\infty} \mathrm{d} \gamma p_{\mathrm{eb}}(v, \gamma) N(\gamma) \\
& =\frac{16 c \sigma_{\mathrm{T}} U_{\mathrm{L}} N_{0}}{27 \pi v_{\mathrm{b}}}\left(v / v_{\mathrm{b}}\right)^{(1-s) / 2} \exp \left(-\left(\frac{v}{\nu_{\mathrm{b}} \gamma_{0}^{2}}\right)^{1 / 2}\right)
\end{aligned}
$$

for frequencies $v>v_{\mathrm{b}}$. At high frequencies the emission is optically thin and the radiation intensities are given by the line-of sight integrals $I_{\text {synch }}(v)=\int_{0}^{\infty} \mathrm{d} l j_{\text {synch }}(v)$ and $I_{\mathrm{eb}}(v)=$ $\int_{0}^{\infty} \mathrm{d} l j_{\mathrm{eb}}(v)$. For ease of exposition we assume constant values of the galactic magnetic field strength, the electrostatic plasma wave energy density and the interstellar electron density so that the characteristic frequencies $v_{\mathrm{s}}$ and $v_{\mathrm{b}}$ will be constants. 


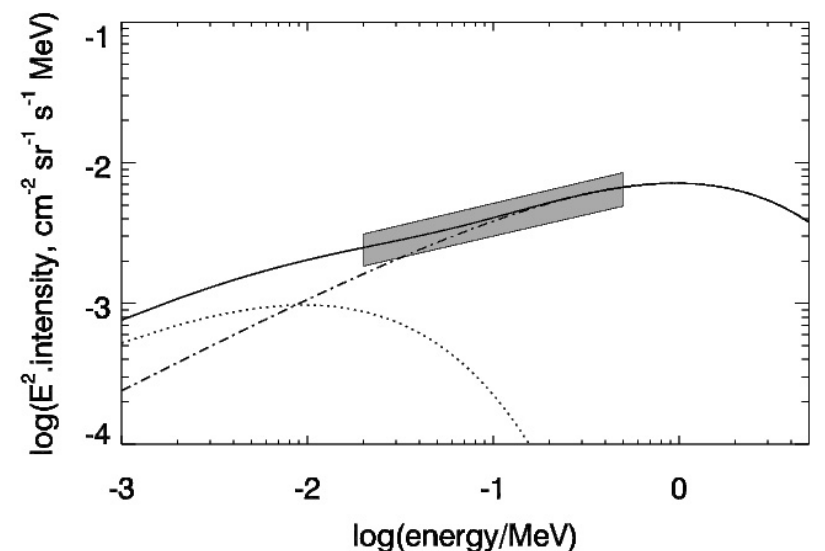

Fig. 1. Comparison of the best fit to the sum (solid line) of the synchrotron and electrostatic bremsstrahlung intensities with the observed diffuse emission (shaded area) from the Galactic disk corrected for the positronium contribution. The shaded area represents the powerlaw fit to the non-positronium component of the diffuse emission for the region $330^{\circ}<l<30^{\circ},-10^{\circ}<b<+10^{\circ}$ given in Strong et al. 2005. The best fit results for the parameter values $v_{\mathrm{b}} / v_{\mathrm{s}} \simeq 100$, $s=1.62, U_{\mathrm{L}}=3.0 U_{B}$ and $\epsilon_{\mathrm{s}}=h v_{\mathrm{s}} \gamma_{0}^{2}=5 \mathrm{keV}$. The dotted line shows the synchrotron flux, the dot-dashed line the electrostatic bremsstrahlung flux.

In Fig. 1 we compare the best fit of the normalised (at $h v=500 \mathrm{keV}$ ) sum of the synchrotron and electrostatic bremsstrahlung intensities with the observed diffuse emission from the inner Galaxy corrected for positronium contributions. The reduced $\chi_{\text {minp.d.f }}^{2}=0.0015$ is obtained for the values $s=1.62, U_{\mathrm{L}} / U_{B}=3.0, v_{\mathrm{b}} / v_{\mathrm{s}}=\epsilon_{\mathrm{b}} / \epsilon_{\mathrm{s}}=100$ and $\epsilon_{\mathrm{s}}=5 \mathrm{keV}$. During the $\chi^{2}$-test we keep the values of the ratio $v_{\mathrm{b}} / \nu_{\mathrm{s}}=100$ and the high-energy synchrotron radiation cutoff $\epsilon_{\mathrm{s}}=5 \mathrm{keV}$ fixed, and only vary the electron spectral index $s$ and the ratio of energy densities $U_{\mathrm{L}} / U_{B}$. The contour intervals of the latter two parameters with different confidence level are shown in Fig. 2.

The value of the high-energy exponential cut-off of the radiatiating electrons $\gamma_{0}$ is determined by requiring that the continuum hard $\mathrm{X}$-ray emission up to $10 \mathrm{keV}$ is predominantly due to synchrotron radiation requiring the value $\epsilon_{s}=$ $h v_{\mathrm{s}} \gamma_{0}^{2}=5 \mathrm{keV}$. Together with the frequency $v_{\mathrm{s}}$ this implies the value $\gamma_{0}=2.7 \times 10^{8}(B / 4 \mu \mathrm{G})^{-1 / 2}$, i.e. maximum electron energies of $10^{14}(B / 4 \mu \mathrm{G})^{-1 / 2} \mathrm{eV}$ which compare favorably well with the maximum energy of shock accelerated electrons found in 14 individual young shell supernova remnants (Reynolds \& Keohane 1999). This choice then also defines the high-energy cut-off of the electrostatic bremsstrahlung emission $\epsilon_{\mathrm{b}}=h \nu_{\mathrm{b}} \gamma_{0}^{2}$ at about $500 \mathrm{keV}$.

The relative $\mathrm{X}$-ray $(<10 \mathrm{keV})$ synchrotron to sub-MeV $(<1 \mathrm{MeV})$ electrostatic bremsstrahlung intensities require a best fit electrostatic plasma wave energy density of $U_{\mathrm{L}}=3 U_{B}$ of about three times the magnetic field energy density. Figure 2 indicates that with 99 percent confidence the average galactic energy density in electrostatic waves is greater than 80 percent of the average galactic magnetic field energy density.

These high-energy primary electrons will also inverse Compton scatter any additional target photons in the inner

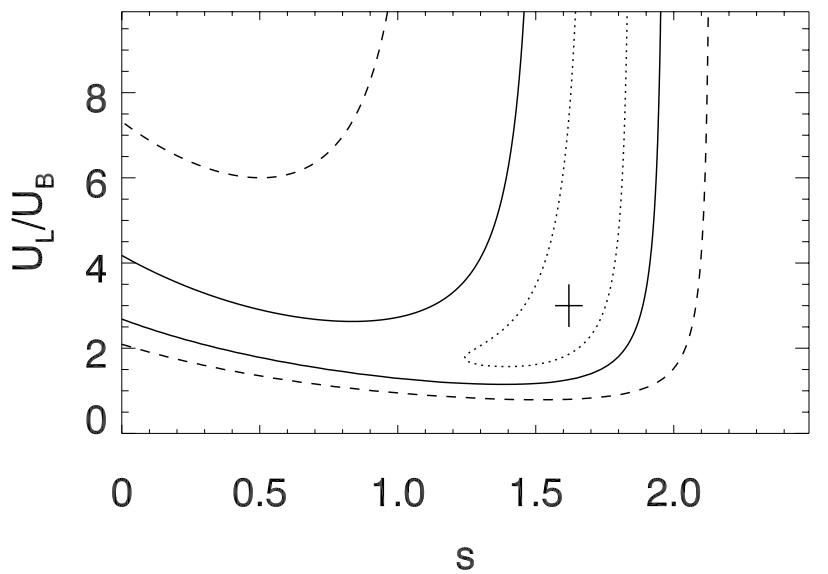

Fig. 2. 20 percent $(0.25 \sigma), 68$ percent $(1 \sigma)$ and 99 percent $(2.6 \sigma)$ confidence intervals of the free parameters electron spectral index $s$ and energy density ratio $U_{\mathrm{L}} / U_{b}$.

Galaxy as UV, optical, infrared or microwave background photons. Denoting with $U_{\mathrm{a}}$ the energy density of any additional photon component, we find in the Thomson limit that the ratio of additional inverse Compton radiation to electrostatic bremsstrahlung at the high-energy cutoff

$\frac{v I_{\mathrm{ICa}}}{v I_{\mathrm{eb}}}=\frac{U_{\mathrm{a}}}{U_{\mathrm{L}}}$

is determined by the respective energy density ratio. For the $2.7 \mathrm{~K}$ microwave background photons we infer

$\frac{v I_{2.7}(30 \mathrm{TeV})}{v I_{\mathrm{eb}}(0.4 \mathrm{MeV})}=3.3$

yielding at $30 \mathrm{TeV} v I_{2.7}(30 \mathrm{TeV}) \simeq 0.003 \mathrm{MeV} \mathrm{s}^{-1} \mathrm{~cm}^{-3} \mathrm{rad}^{-1}$ from the inner Galaxy. At EGRET energies ( $10 \mathrm{GeV})$ this contribution amounts about

$$
\begin{aligned}
v I_{2.7}(10 \mathrm{GeV}) & =0.003\left(\frac{10 \mathrm{GeV}}{30 \mathrm{TeV}}\right)^{0.75} \\
& =7.4 \times 10^{-6} \mathrm{MeV} \mathrm{s}^{-1} \mathrm{~cm}^{-3} \mathrm{rad}^{-1}
\end{aligned}
$$

which is three orders of magnitude smaller than the observed flux from the inner Galaxy (Schlickeiser et al. 1997). Even higher energy densities of optical and infrared photons in the inner part of the Galaxy would not contradict the flux observed by EGRET.

\section{Discussion and summary}

Recent INTEGRAL observations have confirmed the existence of diffuse sub-MeV radiation from the inner Galaxy. Here we have presented a new explanation for the origin of this diffuse component in terms of electrostatic bremsstrahlung radiation from high-energy electrons. This electrostatic bremsstrahlung interpretation requires a very flat power law energy spectrum of high-energy electrons in the inner Galaxy which is reasonable if many point sources with significant spectral dispersion contribute to the injection of high-energy electrons in the Galaxy. The electrostatic bremsstrahlung interpretation avoids the enormous source power problem that previous explanations 
by radiation from low-energy electrons have, and relies on the existence of interstellar electrostatic plasma waves probably generated by the interaction of supernova outflows with the ambient interstellar medium. Our excellent best fit to the observed diffuse emission from the inner Galaxy indicates that the averaged galactic energy density of interstellar electrostatic plasma waves is greater than 80 percent of the averaged galactic magnetic field energy density. The maximum energy of the radiating electrons is about $10^{14} \mathrm{eV}$. Our proposed interpretation as radiation from high-energy electrons then implies a rather structured angular distribution of this radiation component, as the radiating high-energy electrons have small radiative lifetimes and therefore do not propagate very far away from their sources. For an average spatial diffusion coefficient of $D(E) \simeq 10^{27}(E / \mathrm{GeV})^{0.3} \mathrm{~cm}^{2} \mathrm{~s}^{-1}$ and the radiative loss time $t_{s}$ the mean distance travelled by an $10^{14} \mathrm{eV}$ electron is about $\langle x\rangle=\sqrt{2 D t_{\mathrm{s}}} \simeq 30 \mathrm{pc}$ corresponding to 0.2 dregrees if located near the Galactic center. Alternatively, by measuring $\langle x\rangle$ at different photon energies one may determine the value of the average spatial diffusion coefficient $D(E)$.

Acknowledgements. R. Schröder and R. Schlickeiser acknowledge support by the Deutsche Forschungsgemeinschaft through grant Schl 201/16-1.

\section{References}

Brecher, K., \& Burbidge, G. R. 1972, ApJ, 174, 253

Büsching, I., Pohl, M., \& Schlickeiser, R. 2001, A\&A, 377, 1056
Büsching, I., Kopp, A., Pohl, M., et al. 2005, ApJ, 619, 314

Chiuderi, C., \& Veltri, P. 1974, A\&A, 30, 265

Clark, D. H., \& Caswell, J. L. 1976, MNRAS, 174, 267

Dogiel, V. A., Schönfelder, V., \& Strong, A. W. 2002, A\&A, 382, 730

Göbel, W., Hirth, W., \& Fürst, E. 1981, A\&A, 93, 43

Kinzer, R. L., Purcell, W. R., \& Kurfess, J. D. 1999, ApJ, 515, 215

Lebrun, F., Terrier, R., Bazzano, A., et al. 2004, Nature, 428, 293

Mörsberger, U., \& Schlickeiser, R. 1997, Proc. 25th Int. Cosmic Ray Conf. (Durban), vol. 3, 121

Pohl, M., \& Esposito, J. 1998, ApJ, 507, 327

Pohl, M., Lerche, I., \& Schlickeiser, R. 2002, A\&A, 383, 309

Reynolds, S. P., \& Keohane, J. W. 1999, ApJ, 525, 368

Schlickeiser, R. 1997, A\&A, 319, L5

Schlickeiser, R., Pohl, M., Ramaty, R., \& Skibo, J. G. 1997, Proc. 4th Compton Symp., ed. C. D. Dermer, M. S. Strickman, \& J. D. Kurfess (New York: AIP), 449

Schlickeiser, R., Vainio, R., Böttcher, M., et al. 2002, A\&A, 393, 69

Schlickeiser, R. 2003, A\&A, 410, 397

Skibo, J. G., \& Ramaty, R. 1993, A\&AS, 97, 145

Skibo, J. G., Ramaty, R., \& Purcell, W. R. 1996, A\&AS, 120, 403

Strong, A. W., Bennett, K., Bloemen, H., et al. 1994, A\&AS, 292, 82

Strong, A. W., Bouchet, L., Diehl, R., et al. 2003, A\&A, 411, L447

Strong, A. W., et al. 2004, Proc 5th INTEGRAL Workshop, ESA SP-552, 507

Strong, A. W., et al. 2005, A\&A, in press

Strong, A. W., Moskalenko, I. V., \& Reimer, O. 2000, ApJ, 537, 763

Yamasaki, N. Y., Ohashi, T., Takahara, F., et al. 1997, ApJ, 481, 821

Windsor, R. A., \& Kellogg, P. J. 1974, ApJ, 190, 167 